\author{
Military Technical College \\ Kobry El-Kobbah, \\ Cairo, Egypt
}

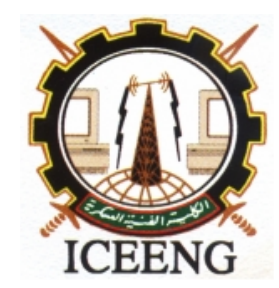

\section{$7^{\text {th }}$ International Conference on Electrical Engineering ICEENG 2010}

\title{
Design of Active Stabilization Controller for a Tactical Rifle
}

\author{
Gamal A.Elnashar * \\ By \\ Abstract:
}

This paper describes an inertial stabilized rifle where a recurve actuator, constructed from piezoelectric material, is used to internially stabilize the barrel assembly of a tactical rifle to compensate for the small user-induced disturbances. This system is an active stabilization system designed to decouple the shooter's interruption effects from the barrel movement based on control systems. Based on the feedback of the targeting error from the sensors, a control system will calculate the desired displacement and force needed to cancel out the human disturbances that are imparted to the rifle. The actuators are designed to cancel out jitter disturbances in a frequency range of $0 \mathrm{~Hz}-5 \mathrm{~Hz}$ It presents research to help protect and increase the defenses of soldiers on ground. While in battle these soldiers' defenses and performance suffer due the intense stressors of combat. A different method for mitigating the depredating physiological effects of a soldier's marksmanship due to battle stressors can be achieved through the design and implementation of active stabilization system for small arms weapons. Combat stress may be defined as the perception of an imminent threat of serious personal injury or death, or the stress of being tasked with the responsibility to protect another party from imminent and serious injury or death, under conditions where response time is minimal. Physiological effects that result from combat include, but are not limited to, a dramatic increase in heart rate, heavy breathing, muscle tremors, and anxiety.

The requirements of this system are discussed and the actuator controller are derived. This research uses pole-placement control techniques to develop control algorithms for simulation. The level of performance for the control algorithm is based on how well measure up to the criteria developed from the rifle.

\section{Keywords:}

Generic rifle, active stabilize, physiological effects, pole placement, Piezoelectric Actuators

* Egyptian Armed Forces 


\section{Introduction:}

Effective fighting tools borne of the application of today's latest technology are needed more than ever to maintain the nation strategic advantage and protect the country soldier in any line of combat whether it's on water, land, or in the air. This paper presents research to help protect and increase the defenses of infantry soldiers on ground. While in battle these soldiers' defenses and performance suffer due the intense stressors of combat. Good marksmanship is critical to infantry mission effectiveness. In combat there are intense external simulations such as incoming fire, loud noises ...etc. There is fear of the unknown and death, especially with the witnessing of loss of life. Unfortunately, the stress generated by all these pressures produces detrimental physiological effects. Studies have shown that the heart beat of a soldier in combat is around 300 beats per minute. (In comparison, Olympic athletes during competition rarely exceed $200 \mathrm{bpm}$ )[1]. In addition to this, the soldiers breathing and muscle shake increases, significantly reducing accuracy. This lost accuracy severely reduces the chance of soldier survival, reduces mission effectiveness and increases collateral damage and civilian casualties. These stressors of combat are well known to the military. Various strategies have been developed to mitigate the effects of stressors on the soldier. These methods include: a) physical conditioning to build-up and maintain gross motor skills, physical strength and stamina, b) mental conditioning to better enable the soldier to manage the psychological effects and c) rigorous marksmanship training including range and simulated combat exercises. Currently, all military personnel are trained in marksmanship techniques; however, only a few attain the performance level of expert due to the extreme fine motor skills and physiological control required for such precision shooting. It is also well known that no matter the level of training, in combat, the accuracy of all shooters significantly degrades. In order to improve the soldier's marksmanship performance in combat, a new stabilized rifle system demonstrator is being developed. The inertially stabilized rifle (INSTAR) eliminates aiming error sources by stabilizing barrel assembly, effectively compensating for the small user induced disturbances.

\section{Mathematical Model}

This phase includes the mathematical model which results from the system's equations of motion which are solved for using Newton's second law applied to the rotational inertias of the stock and barrel in the azimuth and elevation direction. It also discusses the actuator's design and how its constraints will be used in the development of the control design. Key to the stabilization of the barrel is the actuation system. The actuator is composed of multiple piezoceramic layers interconnected in series and/or parallel. A voltage applied to the array of layers produces a net push or pull motion resulting in an applied force with a displacement. The unique design of the actuator allows it to provide sufficient force and displacement and satisfy the size constraints of being able to fit into the stock of the rifle, while adding minimal amount of weight to the rifle. A plant design of the rifle dynamics must be developed before control theory may be applied to the system. The plant is a mathematical model that fully captures the dynamics of the rifle while taking into consideration the rifle's response to human interaction. Equations will be used to develop the plant model since these equations fully describe the motions of the rifle being shot by a human in a stationary position [2] 


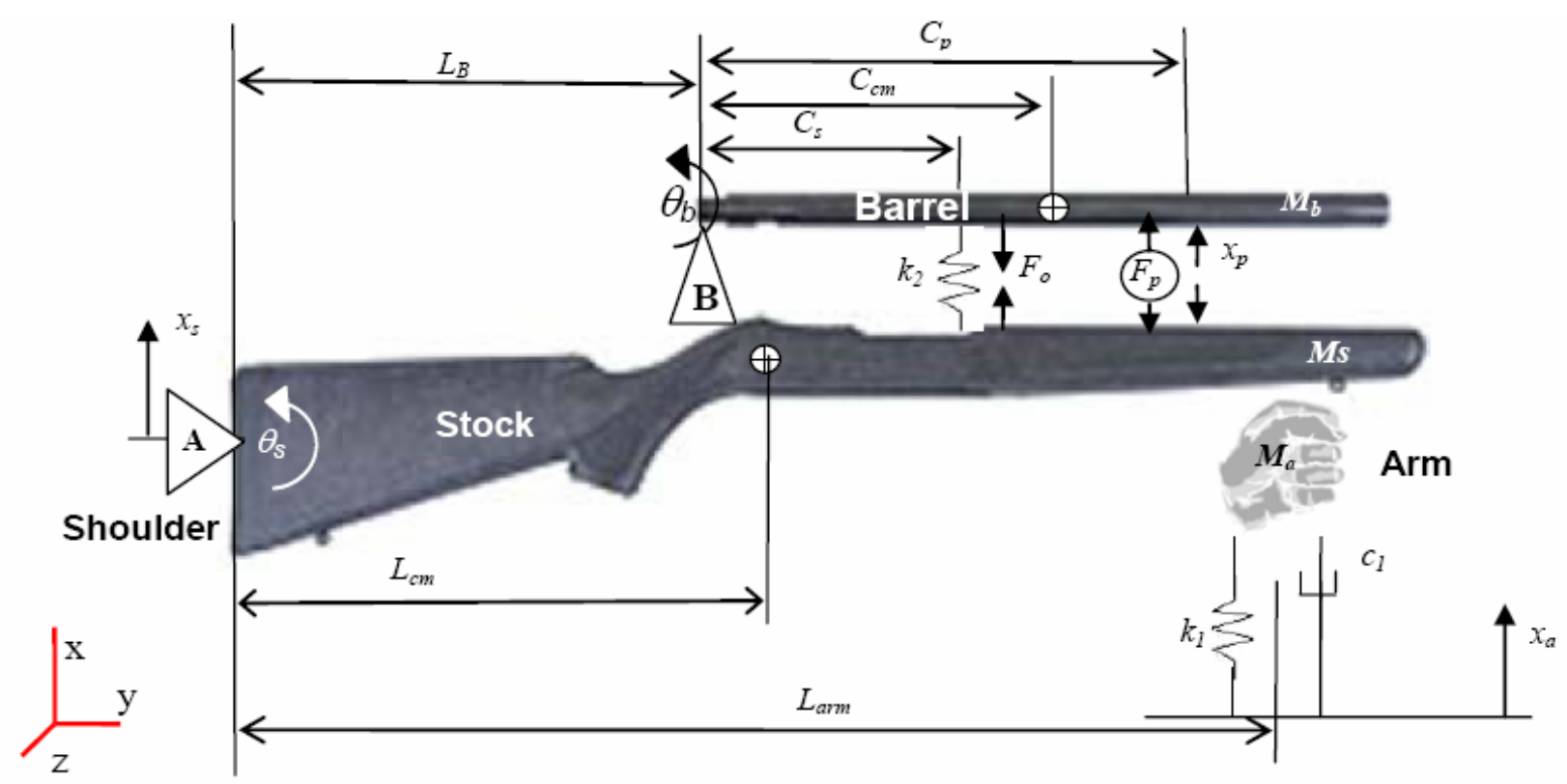

Figure (1): Generic model of a rifle with active stabilization system (Brei et. al, 2003)

Figure(1) is a generic dynamic rifle model. It consists of the stock of the gun, the barrel of the gun, the active suspension stabilizing system, along with the human arm and shoulder disturbance.

The active suspension stabilizing system consists of the actuator which produces a force $\boldsymbol{F}_{\boldsymbol{p}}$ at displacement $\boldsymbol{x}_{p}$, and the preloaded restoring force $\boldsymbol{F}_{\boldsymbol{o}}$ which is produced by spring constant $\boldsymbol{k}_{\boldsymbol{2}}$ which is located between the barrel and the stock of the weapon .

The active suspension stabilizing system serves to buffer out the jitter effects that result from human interaction with the weapon. The actuator moves the barrel of the rifle up and down independent of the stock while spring constant $\boldsymbol{k}_{2}$ produces $\boldsymbol{F}_{\boldsymbol{o}}$ to hold the barrel onto the actuator [2-5].

The barrel and the stock are the two major components of a rifle. Their masses are so large that the masses of the other parts of the rifle are seen as negligible, therefore only the mass of the stock $\boldsymbol{M s}$ and the mass of the barrel $\boldsymbol{M}_{\boldsymbol{b}}$ are placed in the analytical dynamic model . The stock of the rifle serves to facilitate easy steady holding and aiming of the rifle prior to and during firing of the rifle. The barrel of the rifle is a cylindrical metal tube through which the bullet travels before leaving the rifle.

The human disturbances are modeled at the two main points of human-rifle contact with respect to the $x-y-z$ coordinate system shown in Figure 1. These points are where the shoulder and hand touch the rifle. The shoulder disturbance is modeled in Figure (1) with a pivot point of the rifle with an angle $\boldsymbol{\theta}_{\boldsymbol{s}}$ of , and a translation in the positive direction due to shoulder disturbance is modeled as displacement $\boldsymbol{x}_{\boldsymbol{s}}$ at the pivot point A. The arm disturbance is modeled as a mass-spring-damper system to account for the movements due to the musculature of the arm. Figure (1) has two pivot points $\theta_{s}$ the angle of the stock and $\theta_{b}$, the angle of the barrel. Both angles are measured relative to the horizontal, however $\theta_{b}$ is the angle of importance. The angle measured by $\theta_{b}$ corresponds to the targeting error of the rifle system [2-5]. It is desired that $\theta_{b}$ be equal zero. The value of $\theta_{b}$ can be expressed in terms of $\theta_{s}$ and $\boldsymbol{x}_{p}$ as follows:

$\theta_{s}=\theta_{b}-\frac{x_{p}}{C_{P}}$ 
The equations of motion for the rifle were established by applying Newton's second law to the forces in the vertical direction in Figure 2.5. These equations of motion fully describe the motions of the rifle subject to base excitation inputs $\boldsymbol{x}_{\mathrm{a}}, \boldsymbol{x}_{\mathrm{s}}$ and platform force input $\boldsymbol{F p}(\boldsymbol{t})$ [6-8].

$$
M\left[\begin{array}{l}
\ddot{x}_{p} \\
\ddot{\theta} \\
\ddot{\theta}
\end{array}\right]+C\left[\begin{array}{l}
x p \\
\theta
\end{array}\right]+K\left[\begin{array}{l}
x p \\
\theta
\end{array}\right]=G_{s}+G_{a}+H_{p} p+D F_{0}
$$

where the mass matrix $\mathbf{M}$ is

$$
M=\left[\begin{array}{cc}
\frac{-I_{s}+L_{B}^{2} M_{b}}{C_{p}} & I_{s}+L_{B} M_{b}\left(L_{B}+C_{c m}\right) \\
\frac{-M_{c m} C_{B}}{C_{p}} & I_{b} p+M_{b} C_{c m} L_{B}
\end{array}\right]
$$

the damping matrix $\mathbf{C}$ is

the stiffness matrix $\mathrm{K}$ is

$$
C=\left[\begin{array}{cc}
\frac{-C_{\text {arm }} L_{\text {arm }}^{2}}{C_{p}} & C_{\text {arm }} L_{\text {arm }}^{2} \\
0 & 0
\end{array}\right]
$$

$$
K=\left[\begin{array}{cc}
-\left(\frac{C_{s}^{2} k_{2}+k_{1} L_{a r m}^{2}}{C_{p}}\right) & k_{1} L_{\text {arm }}^{2} \\
\frac{k_{2}+C_{s}^{2}}{C_{p}} & 0
\end{array}\right]
$$

the shoulder disturbance matrix $\mathbf{G}_{\mathbf{s}}$ is

$$
G_{S}=\left[\begin{array}{c}
\left(-M_{s} L_{c m} M_{b}\right) \ddot{x}_{S}-L_{a r m} C_{a r m} \dot{x}_{S}-k_{1} L_{a r m} x_{S} \\
\left(-C_{c m} M_{b}\right) \ddot{x}_{S}
\end{array}\right]
$$

the arm disturbance matrix $\mathbf{G}_{\mathbf{a}}$ is

$$
G_{a}=\left[\begin{array}{c}
L_{a r m} C_{a r m} \dot{x}_{s}+k_{1} L_{a r m} x_{a} \\
0
\end{array}\right]
$$

the moment matrix of the actuator is $\mathbf{H}$ 
$H=\left[\begin{array}{c}-C_{p} \\ C_{p}\end{array}\right]$

and the matrix $\mathbf{D}$ represents the moment arms for spring preload $\boldsymbol{F}_{\boldsymbol{0}}$

$$
D=\left[\begin{array}{c}
C_{S} \\
-C_{S}
\end{array}\right]
$$

\section{Actuator}

INSTAR is a tactical rifle designed to address unwanted shooter-induced disturbances by decoupling their effects from the rifle's barrel via an active suspension system [6-8] A free body diagram of the active suspension system including the mass of the rifle is shown in Figure 2 where $W$ is the weight of the barrel. The active suspension system consists of restoring force $\boldsymbol{F}_{s}$ modeled as a spring of stiffness $\boldsymbol{k}_{\boldsymbol{s}}$, an actuator force $\boldsymbol{F}_{a}$ modeled as spring of stiffness $\boldsymbol{k}_{\boldsymbol{a}}$ with an actuator displacement of $\boldsymbol{x}_{\boldsymbol{a}}$, sensors, and a power supply. Distances $L_{w}, L_{s}$ and $L_{a}$ are the moment lengths of $W, \boldsymbol{F}_{s}$, and $\boldsymbol{F}_{a}$, respectively. These moment lengths are measured from the pivot point of the rifle located at the shooter's shoulder. These lengths will be used to derive the equations of motion for the rifle's dynamic model. Based on the feedback of the targeting error $\boldsymbol{\theta}(\mathbf{t})$ from the sensors, a control system will calculate the desired $\boldsymbol{x}_{\boldsymbol{a}}$ and $\boldsymbol{F}_{a}$, needed to cancel out the human disturbances that are imparted to the rifle. The actuators are designed to cancel out jitter disturbances in a frequency range of $0 \mathrm{~Hz}-5 \mathrm{~Hz}$.

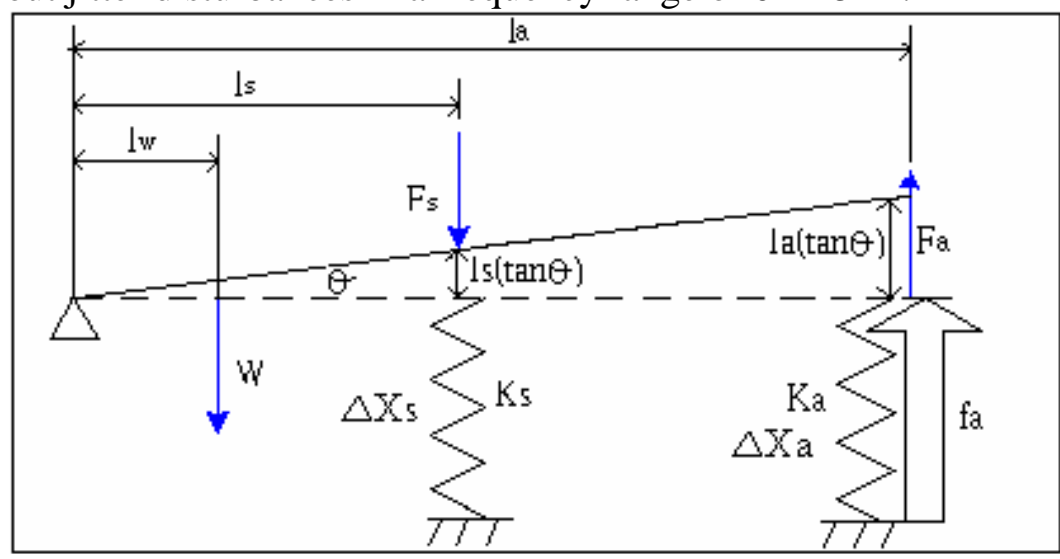

Figure (2): Free Body Diagram Including Shoulder and Arm Disturbance 


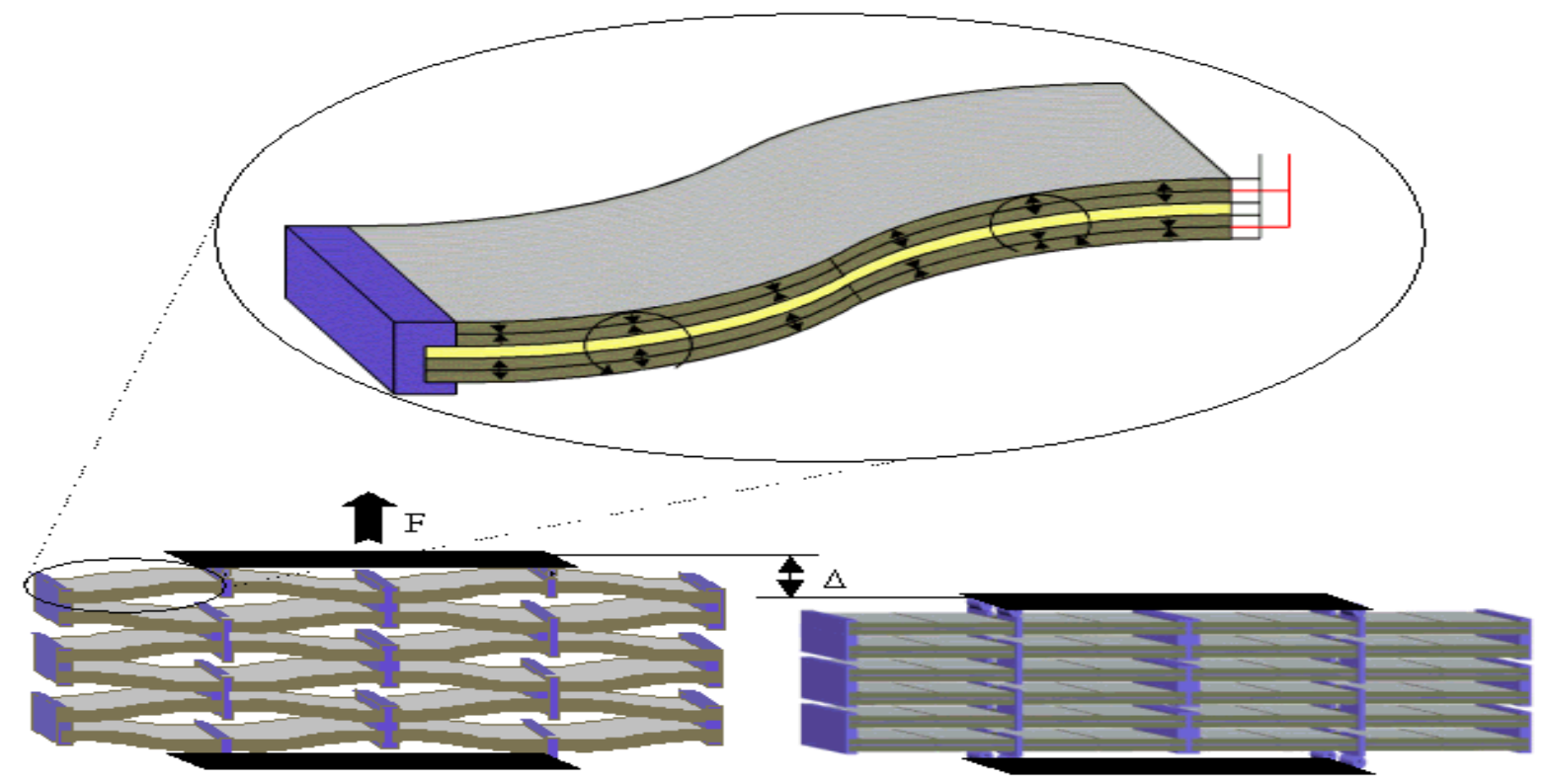

Figure (3): The Actuator's Piezoceramic Layers

The actuator itself is composed of multiple piezoceramic layers interconnected in series and/or parallel. A voltage applied to the array of layers produces a net push or pull motion resulting in an applied force with a displacement as labeled in Figure (3). The unique design of the actuator allows it to provide ample force and displacement and satisfy the size constraints of being able to fit into the stock of the rifle, while adding minimal amount of weight to the rifle [ ].

The actuator moves the barrel in the elevation direction, producing a range of barrel angles $\boldsymbol{\theta}(\mathbf{t})$ The actuator is designed to isolate a shooter induced disturbance from the gun barrel's point of aim for a stationary shooter with targeting error amplitude of 1.5 to 3 silhouettes at various ranges [ ].. If the shooter is located $400 \mathrm{~m}$ from their designated target this equates to a targeting error $\boldsymbol{\theta ( t )}$, of $1.88 \mathrm{mrad}$ as shown in Figure (4).

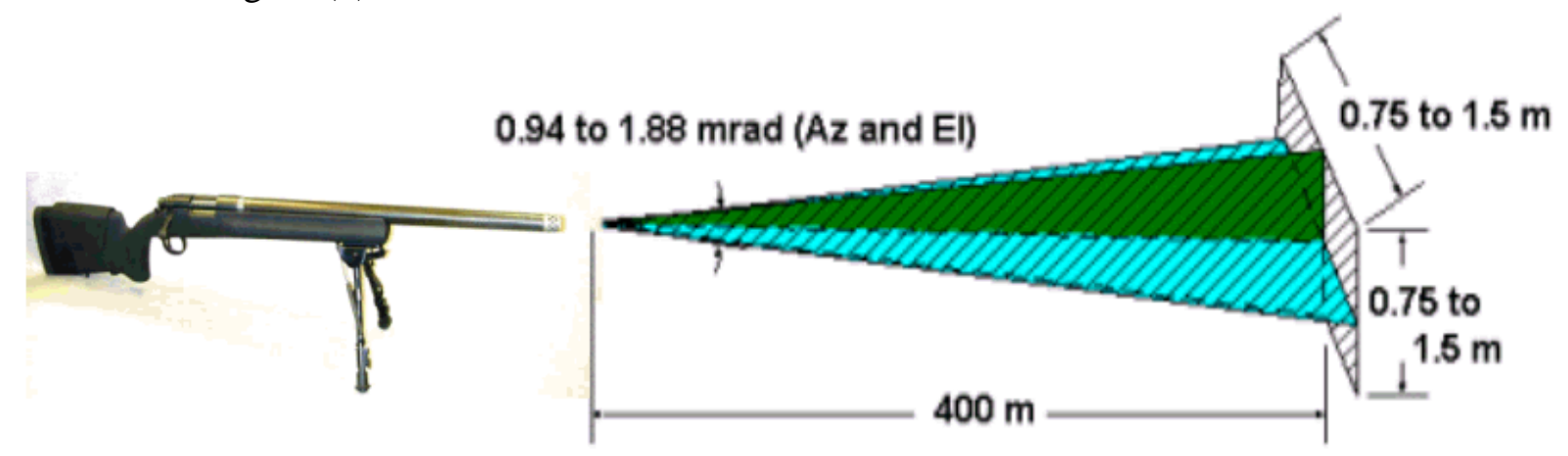

Figure (4): Allowable shooter targeting error range (Brei et. al, 2003)

\section{Static analysis}

Suppose a stationary shooter with gun barrel is interested in hitting a particular point in silhouette area $(0.75 \mathrm{~m} \times 0.75 \mathrm{~m}$ or $1.5 \mathrm{mx} 1.5 \mathrm{~m}$ at the range of $400 \mathrm{~m})$ assuming no wind conditions. It is usually 
assumed that the probability of a particular miss in the range $(x)$ direction is different and independent of the miss in the deflection $(y)$ direction. Given that the distribution in the $\mathrm{x}$ and $\mathrm{y}$ directions are normal and independent. we can write a bivarite PDF as:

$$
f(x, y)=\frac{1}{2 \pi \sigma_{x} \sigma_{y}} \exp \left[\frac{\left(x-v_{x}\right)^{2}}{2 \sigma_{x}^{2}}+\frac{\left(x-v_{y}\right)^{2}}{2 \sigma_{y}^{2}}\right]
$$

This is known as the elliptical form of the distribution because contours of constant $f(x, y)$ are ellipses. We can calculate the probability that $\mathrm{x}$ between two values and $\mathrm{y}$ lies between two other values from cumulative distribution function (CFD) as follows:

$$
\begin{aligned}
& P\left[\left(\begin{array}{lllllll}
X_{1} & \pi & x & \pi & X_{2}
\end{array}\right),\left(\begin{array}{llllll}
Y_{1} & \pi & Y & \pi & Y_{2}
\end{array}\right)\right]
\end{aligned}
$$

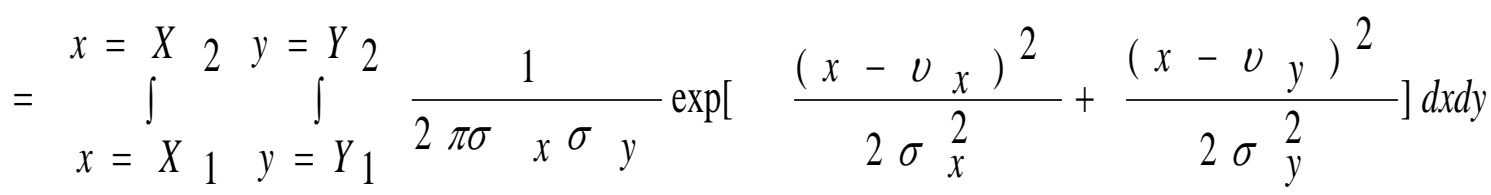

Where $v_{\mathrm{x}}, \sigma_{\mathrm{x}}$ are the mean and standard deviation of the miss distance in the range direction, $v_{\mathrm{y}}, \sigma_{\mathrm{y}}$ are the mean and standard deviation of the miss distance in the deflection direction. This allows us to compute the probability that a particular impact occurs inside a rectangle bordered by $\mathrm{X}_{1}, \mathrm{X}_{2}$ in range direction and $\mathrm{Y}_{1}, \mathrm{Y}_{2}$ in the deflection direction.

\section{Numerical Models}

This section is a layout of the numerical models of the plant and the controllers developed in this paper. It is important that the control system designed to stabilize a tactical rifle meet all design criteria. The fact that the stabilizing system must produce an acceptable output while estimating and controlling the states' values, along with the fact that the control input of the system requires a system of parameters to regulate these performance aspects [8-10]. In the previous literature, we had ignored the presence of disturbances, or noise, in a plant when designing full-state feedback regulators. Designs that ignore noise in a plant are likely to fail when implemented in actual conditions where noise exists. Noise can be divided into two categories: measurement noise or the noise caused by imperfections in the sensors that measure the output variables; and the process noise, or the noise which arises due to ignored dynamics when modeling a plant. Since neither the sensors nor a plant's mathematical model can be perfect, we should always expect some noise in a plant. The uncontrolled system of an assumed tactile rifle is a linear timeinvariant system whose state equation is written as

$$
\begin{gathered}
\dot{x}(t)=A x(t)+B u(t)+F x_{n}(t) \\
y(t)=C x(t)+D u(t)+E x_{n}(t)
\end{gathered}
$$

where $\boldsymbol{x}(\boldsymbol{t})$ is the state-vector of the plant, and $\boldsymbol{x}_{\boldsymbol{n}}(\boldsymbol{t})$ is the noise state- vector where $\boldsymbol{F}$ and $\boldsymbol{E}$ are the noise coefficient matrices in the state and output equations, Respectively. Designing a control system 
using full-state feedback requires that the plant described by Eq. (12) must be controllable, otherwise the control input will not affect all the state variables of the plant. Furthermore, the control law requires that the all the state variables of the system must be measurable, and capable of being fed back to the controller. The controller thus consists of physical sensors, which measure the state variables, and electrical or mechanical devices, called actuators, which provide inputs to the plant based on the desired outputs and the control-law of Eq. (13). Modern controllers invariably use digital electronic circuits to implement the control-law in a hardware. A schematic diagram of the general control system with full-state feedback with noise is shown in Figure (5). and $K$ and Kn are the controller gain matrices

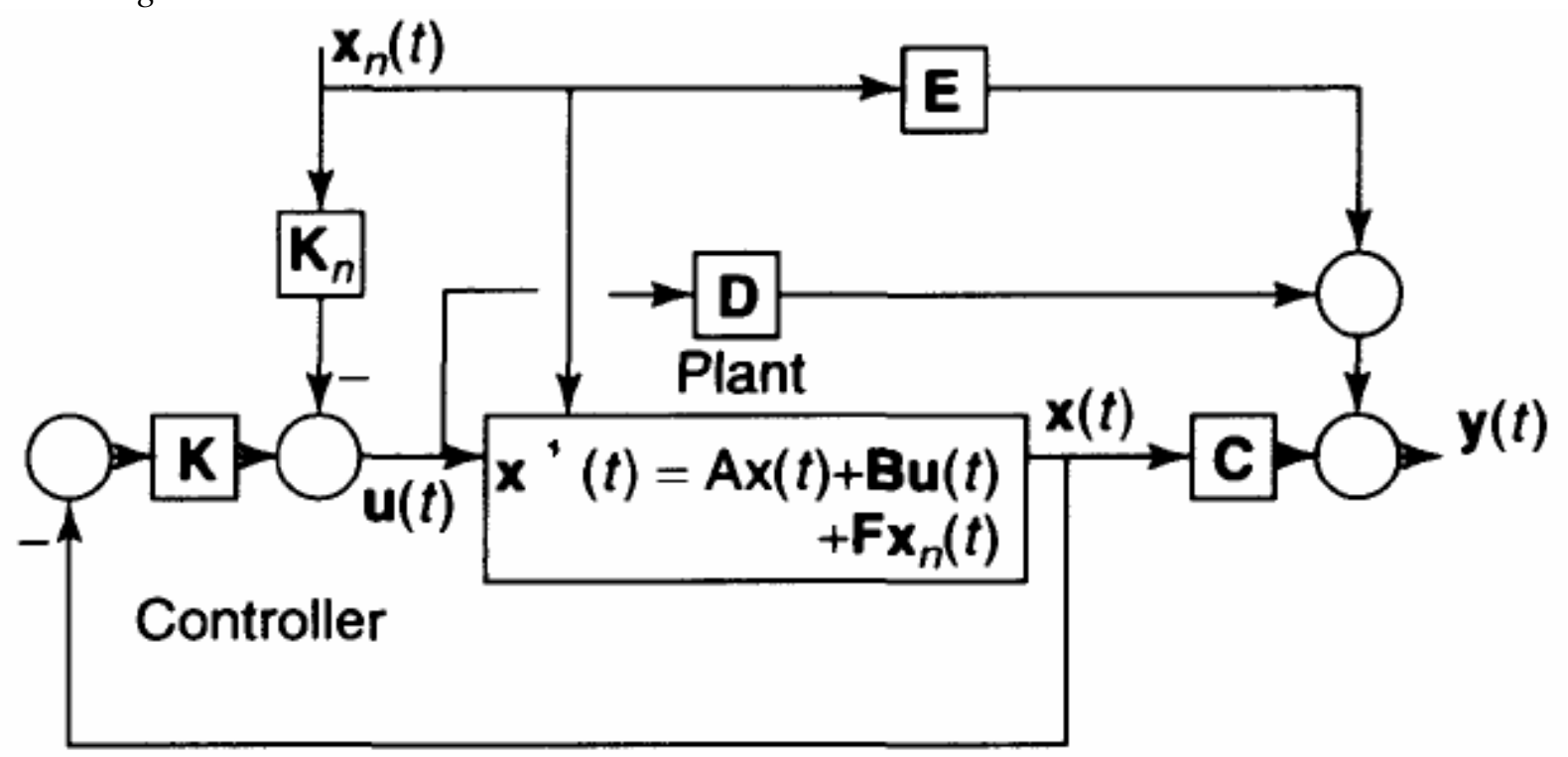

Figure (5): Schematic diagram of full state feedback regulator with noise $\boldsymbol{x}_{n}(\boldsymbol{t})$

To place the closed-loop poles at desired locations while counteracting the effect of the noise, a fullstate feedback regulator is to be designed based on the following control-law:

$$
u(t)=-K x(t)-k_{n} x(t)
$$

By substituting by control law $\boldsymbol{u}(\boldsymbol{t})$ equation into the state equation (12) of the closed loop system, it is written as:

$$
\dot{x}(t)=(A-B K) x(t)+\left(F-B K_{n}\right) x_{n}(t)
$$

Note that Eq. (14) implies that the noise vector, $\boldsymbol{x}_{\boldsymbol{n}}(\boldsymbol{t})$ acts as an input vector for the closed-loop system, whose state-dynamics matrix is $\boldsymbol{A}_{\boldsymbol{C} L}=(\boldsymbol{A}-\boldsymbol{B K})$. An analytical numerical dynamic model for the assault rifle is shown in equation (15). Kinematically it represents a different case from the generic model in Figure (1) in that its stock and barrel are rigidly attached, with the actuator held directly by the arm. Since the stock and barrel are rigidly attached their masses are summed together into and the stock mass is set to zero. A new derivation of equation (2) will be as the following:

$$
\left[\begin{array}{c}
. . \\
x_{p} \\
\ddot{\theta}
\end{array}\right]=(-1)\left[\begin{array}{cc}
a & b \\
0 & 0
\end{array}\right]\left[\begin{array}{c}
\cdot \\
x_{p} \\
\dot{\theta}
\end{array}\right]+(-1)\left[\begin{array}{ll}
e & f \\
g & 0
\end{array}\right]\left[\begin{array}{l}
x_{p} \\
\theta
\end{array}\right]+\left[\begin{array}{l}
h \\
i
\end{array}\right] F_{p}(t)+\left[\begin{array}{cc}
j & k \\
0 & l
\end{array}\right]\left[\begin{array}{l}
d_{1}(t) \\
d_{2}(t)
\end{array}\right]
$$


Where

$$
\begin{aligned}
& \left.\begin{array}{l}
a=\frac{c_{1}}{m_{a}}, b=\frac{-c_{1} l_{p}}{m_{a}}, e=\frac{I_{A}\left(k_{1}+k_{2}\right)+m_{a} l_{p}^{2} k_{2}}{I_{A} m_{a}}, f=\frac{-k_{1} l_{p}}{m_{a}} \\
g=\frac{k_{2} l_{p}}{I_{A}}, h=\frac{I_{A}+m_{a} l_{p}^{2}}{I_{A} m_{a}}, i=\frac{l_{p}}{I_{A}}, j=\frac{-1}{m_{a}}, k=\frac{l_{p}}{I_{A}}, l=\frac{1}{I_{A}}
\end{array}\right\} \\
& d_{1}=-k_{1} x_{a}-c_{1} \dot{x}_{a}-m_{a} \ddot{x}_{s}-c_{1} \dot{x}_{s}-k_{1} x_{s}=A_{1} \sin \left(\omega_{1} t\right) \\
& d_{2}=-m_{r} l_{c m} \ddot{x}_{s}=A_{2} \sin \left(\omega_{1} t\right)
\end{aligned}
$$

To model equation (16) into a state space model the state variables are defined as

$$
\begin{aligned}
& x_{1}=x_{p}, x_{2}=\dot{x}_{p}, y_{1}=\theta, y_{2}=\dot{\theta}, \quad \dot{x}_{1}=x_{2} \\
& \dot{x}_{2}=-a\left(x_{2}\right)-b\left(y_{2}\right)-e\left(x_{1}\right)-f\left(y_{1}\right)+h\left(F_{p}(t)\right)+j\left(d_{1}(t)\right)+k\left(d_{1}(t)\right. \\
& \dot{y}_{1}=y_{2}, \dot{y}_{2}=-g\left(x_{1}\right)+i\left(F_{p}(t)\right)+l\left(d_{2}(t)\right)
\end{aligned}
$$

A plant design of the rifle dynamics must be developed before control theory may be applied to the system. The plant is a mathematical model that fully captures the dynamics of the rifle while taking into consideration the rifle's response to human interaction. These equations will be used to develop the plant model since these equations fully describe the motions of the rifle being shot by a human in a stationary position. Under fully defined parameters, the desired form of the system is:

$$
\begin{aligned}
& {\left[\begin{array}{l}
\dot{x}_{1}(t) \\
\dot{x}_{2}(t) \\
\dot{y}_{1}(t) \\
\dot{y}_{2}(t)
\end{array}\right]\left[\begin{array}{cccc}
0 & 1 & 0 & 0 \\
-48000 & -300 & 16000 & 200 \\
0 & 0 & 0 & 1 \\
-1400 & 0 & 0 & 0
\end{array}\right]\left[\begin{array}{l}
x_{1}(t) \\
x_{2}(t) \\
y_{1}(t) \\
y_{2}(t)
\end{array}\right]+\left[\begin{array}{c}
0 \\
0.2 \\
0 \\
0.3
\end{array}\right] F_{p}(t)+\left[\begin{array}{cc}
0 & 0 \\
0.16 & 0.32 \\
0 & 0 \\
0 & 0.48
\end{array}\right]\left[\begin{array}{l}
d_{1}(t) \\
d_{2}(t)
\end{array}\right],} \\
& \theta(t)=\left[\begin{array}{llll}
0 & 0 & 1 & 0
\end{array}\right]\left[\begin{array}{l}
x_{1}(t) \\
x_{2}(t) \\
y_{1}(t) \\
y_{2}(t)
\end{array}\right]
\end{aligned}
$$

The system represented by equation (18) outputs the barrel angle $\boldsymbol{\theta}(\boldsymbol{t})$. Equation (18) is a fourth order state space dynamic model of a rifle, where the motion is introduced by a shooter's arm and shoulder disturbance, and the actuator force that is required to reduce the amplitude of movement of the rifle's barrel. In equation (18) the state space representation of the rifle's dynamic model, matrix A represents the plant matrix of the rifle which represents the behavior of the states of the system. The state's 
behavior $\boldsymbol{x}_{1}(t)$ that will be closely monitored is which is equal to $\boldsymbol{x}_{p}(t)$, the measurable value of the actuator's displacement. Matrix B in equation (18) represents the behavior of the system's input $\boldsymbol{F}_{p}(t)$. Matrix $C$ in equation is the output matrix of system. It outputs the rifle's barrel angle $\boldsymbol{\theta}(\boldsymbol{t})$. This is also referred to as the targeting error. $\boldsymbol{u}(\boldsymbol{t})$ is the control input which corresponds to the barrel stabilizing actuator force $\boldsymbol{F}_{p}(t) . \boldsymbol{d}_{\boldsymbol{l}}(t)$ is a combination of arm disturbance and shoulder disturbance . $\boldsymbol{d}_{2}(t)$ is only composed of shoulder disturbance. When the shooter holds the gun, ergonomic disturbances cause the gun barrel to oscillate. Disturbances $\boldsymbol{d}_{1}(t)$ and $\boldsymbol{d}_{2}(t)$ are assumed to be sinusoidal with different amplitudes. Upon getting into position and acquiring the target, the shooter activates the control system. Within a short amount of time the control system acts to cancel out the jitters imparted on the rifle barrel by the shooter. This action dramatically increases the accuracy of the shooter. The time from which the shooter activates the control system to when the shooter actually pulls the trigger has to be done in $1 \mathrm{~s}$. This is called Time To Trigger Pull $\left(\mathrm{T}^{3} \mathrm{P}\right)$. Maintaining a $\mathrm{T}^{3} \mathrm{P}$ of $1 \mathrm{~s}$ or less preserves the battery life of the active stabilization system, enabling the system to operate for thousands of shots. The maximum magnitude of actuator force is should be taken in the consideration. This corresponds to a control input range of control signal $F_{p}(t)=u(t)$ to get the maximum actuator displacement $x_{p}(\mathrm{t})$ and save the power usage. In addition to respond in minimum time to trigger, reject disturbances and minimum weight. All control designs for the rifle must meet this constraint to prevent saturation of the actuator.

\section{Results}

This section discusses and presents the simulation results of the designed controlled system using Matlab software which is used to create and test all designed control systems. The plant model including human induced disturbances was designed in section 2 . The disturbances are $3 \mathrm{~Hz}$ sinusoidal waves with various amplitudes. The amplitudes were modified to result in the allowable plant targeting error of $\boldsymbol{\theta}(\boldsymbol{t})=3 \mathrm{mrad}$. Figure $(6)$ simulates the time response from $\mathrm{t}=0$ to the final time $\mathrm{t}=3 \mathrm{sec}$ with

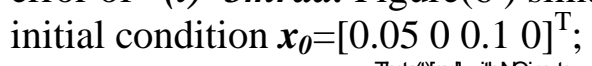
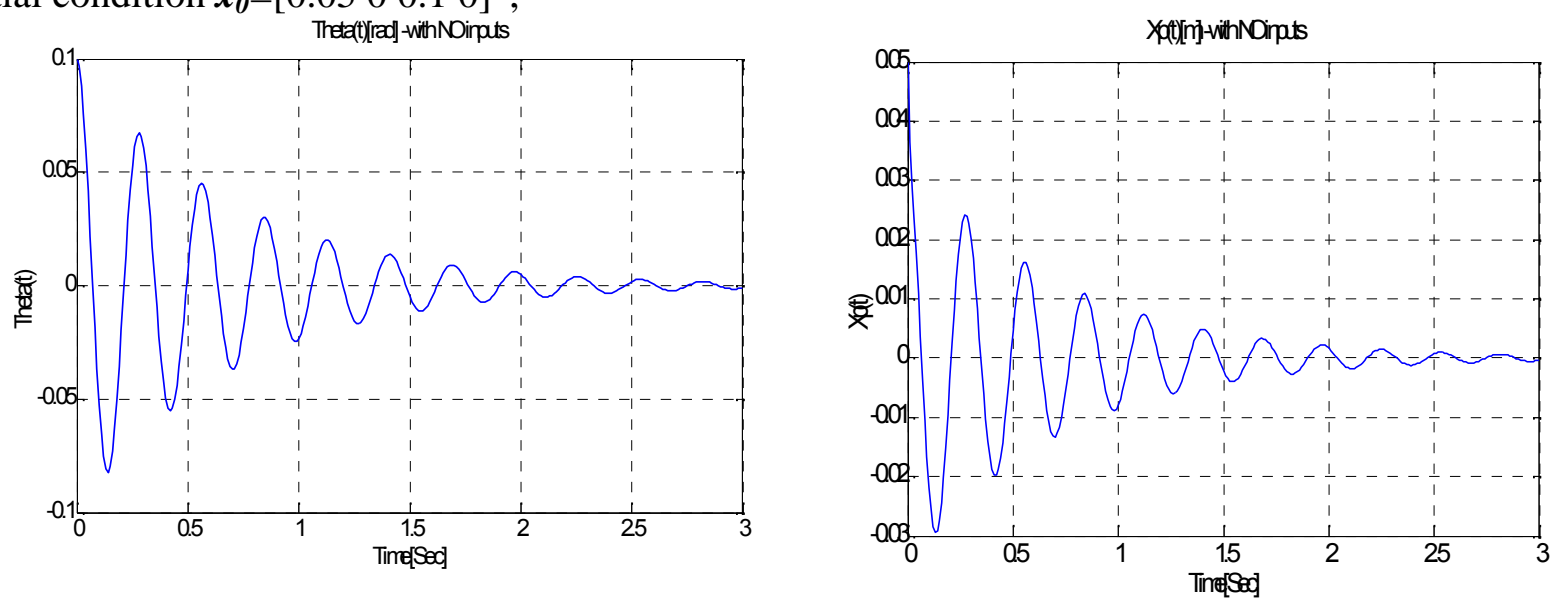

Figure (6): Target error and displacement for uncontrolled system with initial condtion only

The rifle plant model along human disturbances produces a targeting error $\boldsymbol{\theta}(\boldsymbol{t})=\mathbf{3} \boldsymbol{m r a d}$ which writhen the limits of the targeting error distribution allowed by the constraint of the actuator displacement 
magnitude $\boldsymbol{x}_{\boldsymbol{p}}(\mathrm{t})$. Figure (7) displays the dynamic additional behavior of $\theta(t)$ and $\boldsymbol{x}_{\boldsymbol{p}}(\mathrm{t})$ for human disturbances and the actuator force $\boldsymbol{F}_{\boldsymbol{p}}(\boldsymbol{t})=0$ for the uncontrolled system.
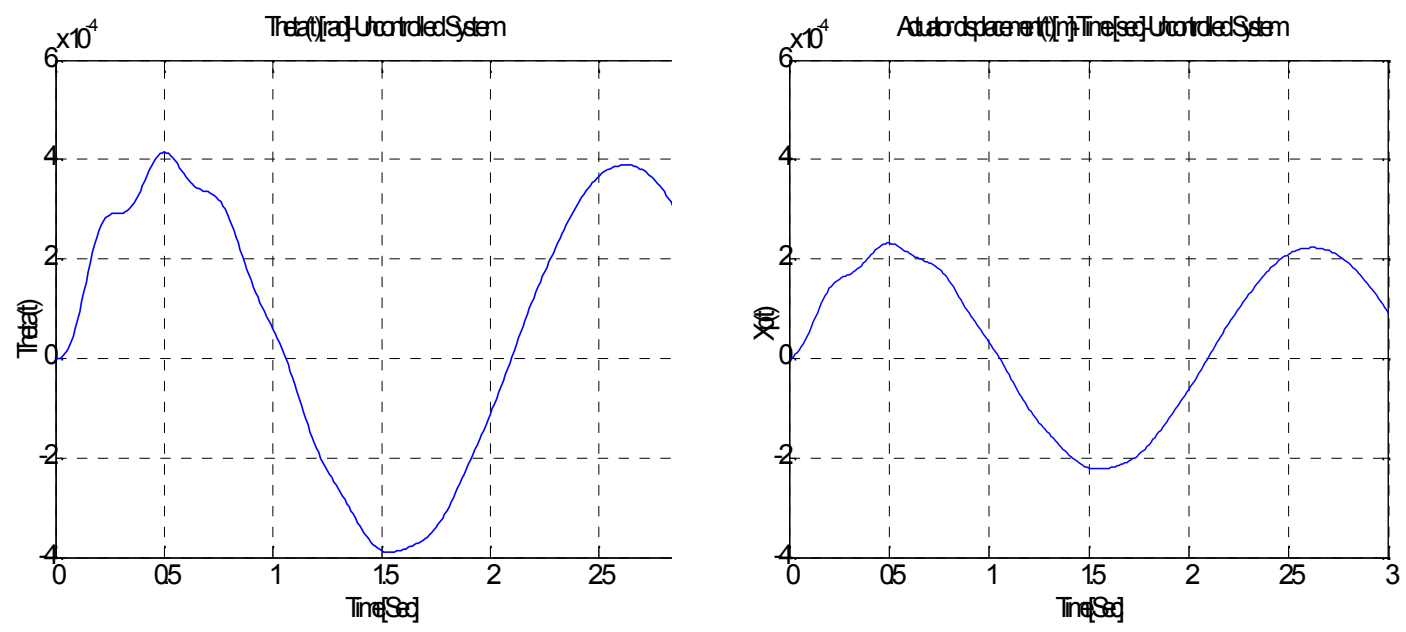

Figure (7): Target error and displacement for uncontrolled system due to disturbancies

The regulator feedback gain matrix, $\boldsymbol{K}$, is selected, firstly, to place the closed loop poles (eigenvalues of $\left.\boldsymbol{A}_{C L}\right)$ at desired locations. While we may not know the exact process by which the noise, $\boldsymbol{x}_{n}(\boldsymbol{t})$, is generated (because it is usually a stochastic process,), we can develop an approximation of how the noise affects the plant by deriving the noise coefficient matrix, $\boldsymbol{F}$, from experimental observations. Once $\boldsymbol{F}$ is known reasonably, the regulator noise gain matrix, $\boldsymbol{K}_{\boldsymbol{n}}$, can be selected such that the effect of the noise vector, $\boldsymbol{x}(\boldsymbol{t})$, on the closed-loop system is minimized. It would, of course, be ideal if we can make $\left(\boldsymbol{F}-\boldsymbol{B} \boldsymbol{K}_{n}\right)=\boldsymbol{0}$, in which case there would be absolutely no influence of the noise on the closedloop system. However, it may not be always possible to select the (rq) unknown elements of $\boldsymbol{K}_{\boldsymbol{n}}$ to satisfy the $(\boldsymbol{n q})$ scalar equations constituting $\left(\boldsymbol{F}-\boldsymbol{B} \boldsymbol{K}_{\boldsymbol{n}}\right)=\boldsymbol{0}$, where $\boldsymbol{n}$ is the order of the plant, $\boldsymbol{r}$ is the number of inputs, and $\boldsymbol{q}$ is the number of noise variables in the noise vector $\boldsymbol{x}_{\boldsymbol{n}}(\boldsymbol{t})$. When $\boldsymbol{r}<\boldsymbol{n}$ (as it is usually the case), the number of unknowns in $\left(\boldsymbol{F}-\boldsymbol{B} \boldsymbol{K}_{n}\right)=\boldsymbol{0}$ is less than the number of scalar equations, and hence all the equations cannot be satisfied. If $\boldsymbol{r}=\boldsymbol{n}$, and the matrix $\boldsymbol{B}$ is non-singular, then we can uniquely determine the regulator noise gain matrix by $\boldsymbol{K}_{\boldsymbol{n}}=\boldsymbol{B}^{-1} \boldsymbol{F}$. In the rare event of $\boldsymbol{r}>\boldsymbol{n}$, the number of unknowns exceed the number of equations, and all the equations, $\left(\boldsymbol{F}-\boldsymbol{B} \boldsymbol{K}_{\boldsymbol{n}}\right)=0$, can be satisfied by appropriately selecting the unknowns, though not uniquely.

The plant of equation(18) is stable as the eigenvalues located at $-1.41+22.3 \mathrm{i},-1.41-22.3 \mathrm{i}, 149+157 \mathrm{i}$, and $149-157 \mathrm{i}$ with damping 0.0634 and 0.688 and with frequency 22.3 and $216 \mathrm{rad} / \mathrm{sec} \mathrm{respectively.}$ Also, the system is controllable, implying pole-placement is possible. The regulator feedback gain is obtained $\mathrm{K}=10^{5}$ [7.06 .0163 2.025.019] for desired poles [-200-0.5i -200+0.5i -400+0.5i -400-0.5i]. Figure (8) shows target error and displacement for reglator system. It leading to required performance as .To determine remaining regulator matrix, $\boldsymbol{K}_{\boldsymbol{n}}=\left[\begin{array}{ll}K_{n 1} & K_{n 2}\end{array}\right]$, let us look at the matrix $\left(\boldsymbol{F}-\boldsymbol{B} \boldsymbol{K}_{\boldsymbol{n}}\right)$ :

$$
F-B K_{n}=\left[\begin{array}{cc}
0 & 0 \\
0.162-0.197 k_{n 1} & 0.32-0.197 k_{n 2} \\
0 & 0 \\
-0.327 k_{n 1} & 0.478-0.323 k n 2
\end{array}\right]=\left[\begin{array}{cc}
0 & 0 \\
0 & 0 \\
0 & 0 \\
-0.268 & -.0478
\end{array}\right]
$$


This equation tells us that it is impossible to make all elements of $\left(F-B K_{n}\right)$ zeros, by selecting the two unknowns. Designs parameters, $K_{n 1}, K_{n 2}$. The next best thing to $\left(F-B K_{n}\right)=0$ is making largest elements of $\left(F-B K_{n}\right)$ zeros, and living with the other non-zero elements.

With $\left(F-B K_{n}\right)$ given by Eq. (19), we are always going to have some effect of noise on the closed-loop system, which hopefully, will be small. The most satisfying thing about Eq. (19) is that the closed-loop system given by Eq. (18) is uncontrollable with noise as the input. This means that the noise is not going to affect all the state variables of the closed-loop system.

Let us see by what extent the noise affects our closed-loop design by calculating the system's response with a noise vector, $\boldsymbol{x}_{n}(t)=\left[\begin{array}{ll}A_{1} & A_{2}\end{array}\right] \sin (w t)$ which acts as an input to the closed-loop system given by Eq. (18), with zero initial conditions. Such a noise model is too simple; actual noise is nondeterministic (or stochastic), and consists of a combination of several frequencies, rather than only one frequency ( $3 \mathrm{rad} / \mathrm{sec}$ ) as assumed here. Figure (9) shows Target error and displacement for poleplacement controlled system with with disturbancies, $\boldsymbol{x}_{\boldsymbol{n}}(\boldsymbol{t})$. This leading to desirable performance.
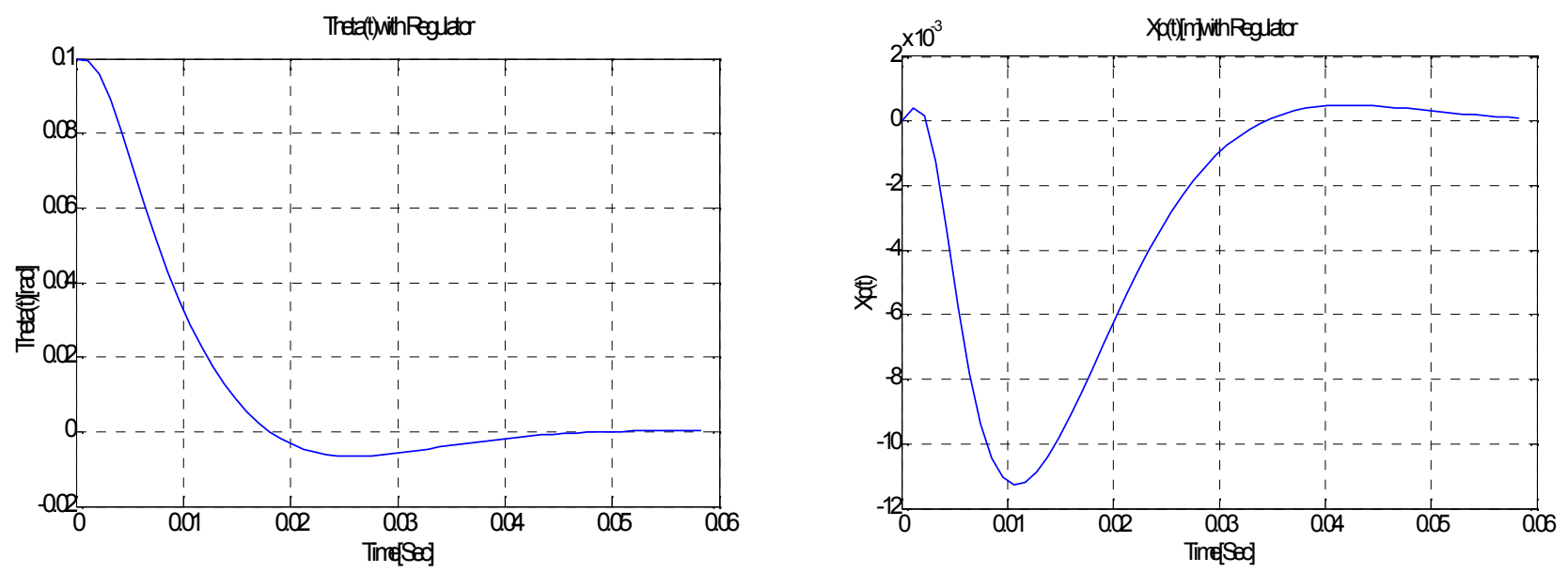

Figure (8): Target error and displacement for pole-placement controlled system
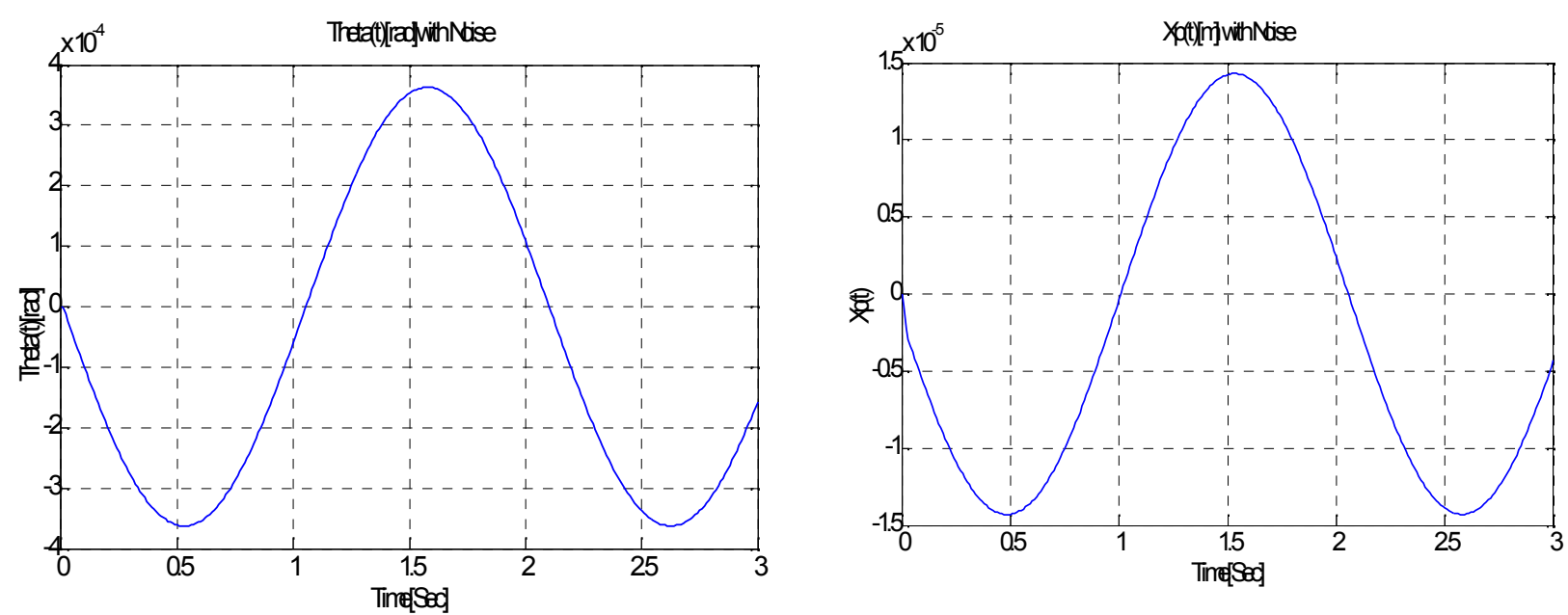

Figure (9):Target error and displacement for pole-placement controlled system due todisturbancies 


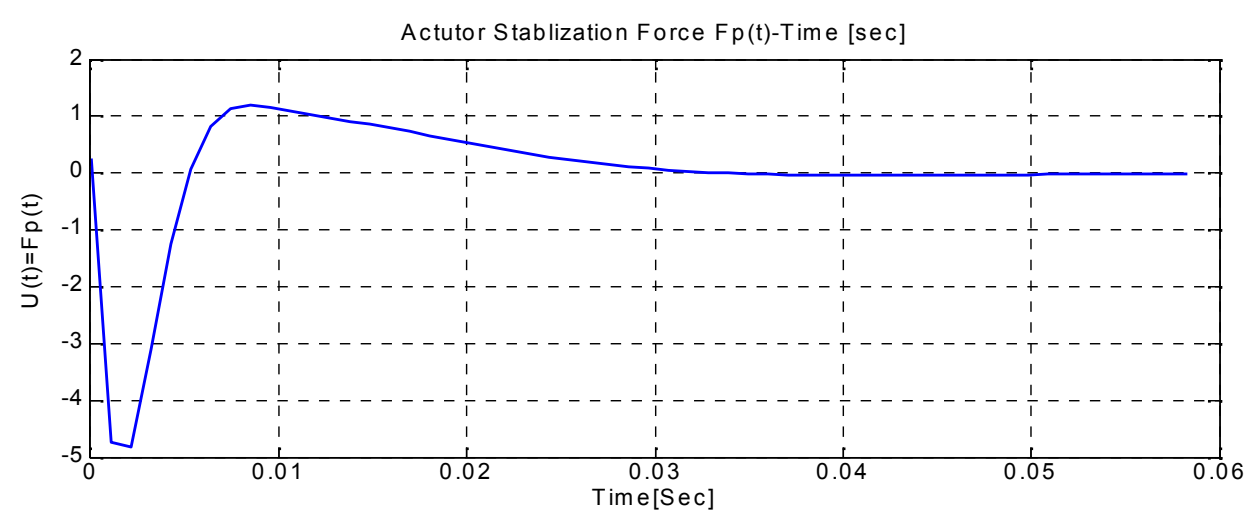

Figure (10): Actuator Force $\mathrm{u}(\mathrm{t})=\mathrm{F}_{\mathrm{p}}(\mathrm{t})$ for controlled system due to disturbancies

Controlling sate values, targeting error, as well as the magnitude of actuator force is main factor to choose the controller. Figure (10) shows the amount of force $u(t)=F_{p}(t)$ required by the actuator to produce the results of Figure(9). The maximum value of force should be taken in the consideration to be under the saturation limit. The amplitude of barrel vibration has significantly been decreased as a result of adding the pole placement with noise controller. It acts as a damper to reduce the jitter, and shorten the system's transient response [11-15].

\section{Conclusions:}

In this paper we analyzed the equations of motion for the rifle stabilization system and its actuator design. Upon review of the control design criteria, pole-placement with noise controllers where designed. The controller handles the task of receiving the rifle's targeting error as input and producing a stabilizing input command to the actuator. The design motivation behind the controllers is to produce a command signal to the actuator that reduces barrel jitter that results from human induced disturbances. This paper described an inertial stabilized rifle where a recurve actuator, constructed from piezoelectric material, is used to internially stabilize the barrel assembly of a tactical rifle to compensate for the small user-induced disturbances. This system is an active stabilization system designed to decouple the shooter's interruption effects from the barrel movement based on control systems. Based on the feedback of the targeting error from the sensors, a control system will calculate the desired displacement and force needed to cancel out the human disturbances that are imparted to the rifle. The actuators are designed to cancel out jitter disturbances in a frequency range of $0 \mathrm{~Hz}-5 \mathrm{~Hz}$ Computer simulations were ran for the controllers in a time interval of 0Sec $<t<3 \mathrm{Sec}$. Within the simulation $3 \mathrm{hz}$ disturbance were induced into each controller design. The controller performed at a level of acceptance. The controllers were successful in minimizing the effects of human shooter disturbances in relation to the shooter's targeting error while meeting that actuator's force and displacement criteria.

This system will lead to greater soldier survivability with less ammunition expended, reduced training requirements and war fighting with less collateral damage. It represents many engineering challenges that can be effectively met using smart structures technology 


\section{References:}

[1] Alejandro Porter White, Control Design for an Inertially Stabilized Rifle, Thesis Virginia Polytechnic Institute and State University, 2007

[2] Brei, D., Luntz, J., and Barnes, B., "Small Arms gun Barrel Stabilization Using High Energy Density, Rugged, and Low Creep Actuators", Specifications Report, University of Michigan, Ann Arbor, MI., June 2005

[3] Lindner, D., Celano, T., and Ide, E., "Vibration Suppression Using a Proofmass Actuator Operating in Stroke/Force Saturation", ASME Journal of Vibration and Acoustics, Vol 113, pp.423-433, 1991

[4] Brei, D., James, V., Lindner, D., Zhu, H., Lavigna, C, "Development and Demonstration of INSTAR-Inertially Stabilized Rifle”, Proceedings of SPIE Conference, 2003

[5] Clymer, A., "The Mechanical Analog Computers of Hannibal Ford and William Newell”, IEEE Annals of the History of Computing, Vol 15, Issue 2, 1993

[6] Pathak, A., Brei, D., Luntz, J., and Lavigna, C.,"A Dynamic Model for Generating Actuator Specifications for Small Arms Barrel Active Stabilization”, University of Michigan \& TechnoSciences, Inc., Ann Arbor, MI \& Lanham, MD. ., 1997

[7] Mattice, M., and LaVigna, C, "Innovative Active Control of Gun Barrels Using Smart Materials”, Proceedings of SPIE Conference, Vol3039, pp630-641, 1997

[8] Clarence W. de Silva, Vibration Damping, Control, and Design, Taylor \& Francis Group, LLC, .2007

[9] Harold Josephs and Ronald L. Huston, Dynamics of mechanical systems , CRC, 2002

[10] John J. D’Azzo and Constantine H. Houpis; LINEAR CONTROL SYSTEM ANALYSIS AND DESIGN WITH MATLAEP, Fifth Edition, 2003

[11] Stanislaw H. Zak, Systems and Control, Oxford University Press, Inc., 2003

[12] Robust Control System Design, Advanced State Space Techniques, Second Edition, Chia-Chi Tsui, by Marcel Dekker, 2004

[13] Nonlinear Control systems, Marcel Dekker, Z Ljubomir, KuljaCa D. MARCELD EKKERIN, C., 2003

[14] M.T. White, M. Tomizuka, and C. Smith. Rejection of disk drive vibration and shock disturbances with a disturbance observer. In Proceedings of the 1999 American Control Conference, pages 4127-4131, San Diego, CA, June 1999

[15] De Silva,. VIBRATION - Fundamentals and Practice, 2nd ed., Taylor \& Francis, CRC Press, 2006 